\title{
MEASURES AND THEIR RANDOM REALS
}

\author{
JAN REIMANN AND THEODORE A. SLAMAN
}

\begin{abstract}
We study the randomness properties of reals with respect to arbitrary probability measures on Cantor space. We show that every noncomputable real is non-trivially random with respect to some measure. The probability measures constructed in the proof may have atoms. If one rules out the existence of atoms, i.e. considers only continuous measures, it turns out that every non-hyperarithmetical real is random for a continuous measure. On the other hand, examples of reals not random for any continuous measure can be found throughout the hyperarithmetical Turing degrees.
\end{abstract}

\section{INTRODUCTION}

Over the past decade, the study of algorithmic randomness has produced an impressive number of results. The theory of Martin-Löf random reals, with all its ramifications (e.g. computable or Schnorr randomness, lowness and triviality) has found deep and significant applications in computability theory, many of which are covered in recent books by Downey and Hirschfeldt [5] and Nies [23. Usually, the measure for which randomness is considered in these studies is the uniform $(1 / 2,1 / 2)$-measure on Cantor space, which is measure theoretically isomorphic to Lebesgue measure on the unit interval.

However, one may ask what happens if one changes the underlying measure. It is easy to define a generalization of Martin-Löf tests which allows for a definition of randomness with respect to arbitrary computable measures. For arbitrary measures, the situation is more complicated. Martin-Löf [20] studied randomness for arbitrary Bernoulli measures, Levin [16-18] studied arbitrary measures on $2^{\omega}$, while Gács 7 ] generalized Levin's approach to a large class of computable metric spaces. Most recently, Hoyrup and Rojas [10] showed that Levin's approach can be extended to any computable metric space. It also turned out that much of the theory (i.e. existence of a universal test, the connection with descriptive complexity, etc.) can be preserved under reasonable assumptions for the underlying space.

In this paper, we study the following question: Given a real $x \in 2^{\omega}$, with respect to which measures is $x$ random? This can be seen as a dual to the usual investigations in algorithmic randomness: Given a (computable) measure $\mu$ (where $\mu$ usually is the uniform distribution), what are the properties of a $\mu$-random real?

Of course, every real $x$ is trivially random with respect to a measure $\mu$ which assigns some positive mass to it (as a singleton set), i.e. $\mu\{x\}>0$. But one can ask if there is a measure for which this is not the case and $x$ is still random. It turns out that this is possible precisely for the non-computable reals. Furthermore, one

Received by the editors February 19, 2008 and, in revised form, May 14, 2013.

2010 Mathematics Subject Classification. Primary 03D32, 68Q30.

The first author was partially supported by NSF grants DMS-0801270 and DMS-1201263.

The second author was partially supported by NSF grants DMS-0501167 and DMS-1001551. 
could ask whether there exists a measure $\mu$ such that $x$ is $\mu$-random and $\mu$ does not have atoms at all, i.e. $\mu\{y\}=0$ for all $y$. The answer to this question shows an unexpected correspondence between the randomness properties of a real and its complexity in terms of effective descriptive set theory: If $x$ is not $\Delta_{1}^{1}$, then there exists a non-atomic measure with respect to which $x$ is random.

These results motivate a further investigation. What is the exact classification of all reals (inside $\Delta_{1}^{1}$ ) which are not random with respect to any continuous measure? If we look at $n$-randomness $(n \geq 2)$, what is the size and structure of the reals that are not $n$-random for some continuous measure? The latter question will be studied in a separate paper [26].

\section{Transformations and measures on Cantor space}

In this section we first quickly review the basic notions of Turing functionals and of measures on Cantor space $2^{\omega}$. The space of probability measures on $2^{\omega}$ is compact Polish, and we can devise a suitable, effective representation of measures in terms of Cauchy sequences with respect to a certain metric. This enables us to code measures as binary sequences so we can use them as oracles in Turing machine computations. This way we can extend Martin-Löf's notion of randomness to arbitrary measures by requiring that a Martin-Löf test for a measure is uniformly enumerable in a representation of the measure. This will be done in Section 3 .

2.1. The Cantor space as a metric space. The Cantor space $2^{\omega}$ is the set of all infinite binary sequences, also called reals. The usual metric on $2^{\omega}$ is defined as follows: Given $x, y \in 2^{\omega}, x \neq y$, let $x \sqcap y$ be the longest common initial segment of $x$ and $y$ (possibly the empty string $\epsilon$ ). Define

$$
d(x, y)= \begin{cases}2^{-|x \sqcap y|} & \text { if } x \neq y, \\ 0 & \text { if } x=y\end{cases}
$$

Endowed with this metric, $2^{\omega}$ is a compact Polish space. A countable basis is given by the cylinder sets

$$
\llbracket \sigma \rrbracket=\{x: x \uparrow|\sigma|=\sigma\},
$$

where $\sigma$ is a finite binary sequence (string), and $|\sigma|$ denotes the length of $\sigma$. We use $2^{<\omega}$ to denote the set of all finite binary sequences, and we use $\sqsubseteq$ to denote the usual prefix partial ordering between finite strings. This partial ordering extends in a natural way to $2^{<\omega} \cup 2^{\omega}$. Thus, $x \in \llbracket \sigma \rrbracket$ if and only if $\sigma \sqsubset x$. Finally, given $U \subseteq 2^{<\omega}$, we write $\llbracket U \rrbracket$ to denote the open set induced by $U$, i.e. $\llbracket U \rrbracket=\bigcup_{\sigma \in U} \llbracket \sigma \rrbracket$.

2.2. Turing functionals. The notion of a Turing functional will be important in this paper, so we give a formal definition and explain how functionals give rise to partial, continuous mappings from $2^{\omega}$ to $2^{\omega}$.

A Turing functional $\Phi$ is a computably enumerable set of triples $(m, k, \sigma)$ such that $m$ is a natural number, $k$ is either 0 or 1 , and $\sigma$ is a finite binary sequence. Further, for all $m$, for all $k_{1}$ and $k_{2}$, and for all compatible $\sigma_{1}$ and $\sigma_{2}$, if $\left(m, k_{1}, \sigma_{1}\right) \in$ $\Phi$ and $\left(m, k_{2}, \sigma_{2}\right) \in \Phi$, then $k_{1}=k_{2}$ and $\sigma_{1}=\sigma_{2}$.

In the following, we will also assume that Turing functionals $\Phi$ are use-monotone, which means the following hold.

(1) For all $\left(m_{1}, k_{1}, \sigma_{1}\right)$ and $\left(m_{2}, k_{2}, \sigma_{2}\right)$ in $\Phi$, if $\sigma_{1}$ is a proper initial segment of $\sigma_{2}$, then $m_{1}$ is less than $m_{2}$. 
(2) For all $m_{1}$ and $m_{2}, k_{2}$ and $\sigma_{2}$, if $m_{2}>m_{1}$ and $\left(m_{2}, k_{2}, \sigma_{2}\right) \in \Phi$, then there are $k_{1}$ and $\sigma_{1}$ such that $\sigma_{1} \sqsubseteq \sigma_{2}$ and $\left(m_{1}, k_{1}, \sigma_{1}\right) \in \Phi$.

We write $\Phi^{\sigma}(m)=k$ to indicate that there is a $\tau$ such that $\tau$ is an initial segment of $\sigma$, possibly equal to $\sigma$, and $(m, k, \tau) \in \Phi$. In this case, we also write $\Phi^{\sigma}(m) \downarrow$, as opposed to $\Phi^{\sigma}(m) \uparrow$, indicating that for all $k$ and all $\tau \sqsubseteq \sigma,(m, k, \tau) \notin \Phi$.

If $x \in 2^{\omega}$, we write $\Phi^{x}(m)=k$ to indicate that there is an $l$ such that $\Phi^{x \uparrow l}(m)=$ $k$. This way, for given $x \in 2^{\omega}, \Phi^{x}$ defines a partial function from $\omega$ to $\{0,1\}$ (identifying reals with sets of natural numbers). If this function is total, it defines a real $y$, and in this case we write $\Phi(x)=y$ and say that $y$ is Turing reducible to $x$ via $\Phi, y \leq_{\mathrm{T}} x$.

By use-monotonicity, if $\Phi^{\sigma}(m) \downarrow$, then $\Phi^{\sigma}(n) \downarrow$ for all $n<m$. This way, $\Phi^{\sigma}$ gives rise to a (possibly infinite) string $\alpha$. If $\Phi^{\sigma}(n) \uparrow$ for all $n$, we put $\alpha=\epsilon$. We write $\Phi(\sigma)=\alpha$. This way a Turing functional induces a function from $2^{<\omega}$ to $2^{<\omega} \cup 2^{\omega}$ that is monotone; that is, $\sigma \sqsubseteq \tau$ implies $\Phi(\sigma) \sqsubseteq \Phi(\tau)$. We can effectively approximate it by prefixes. More precisely, there exists a computable mapping $(\sigma, s) \mapsto \Phi_{s}(\sigma) \in 2^{<\omega}$ so that $\Phi_{s}(\sigma) \sqsubseteq \Phi_{s+1}(\sigma), \Phi_{s}(\sigma) \sqsubseteq \Phi_{s}(\sigma \frown i)(i \in\{0,1\})$, and $\lim _{s} \Phi_{s}(\sigma)=\Phi(\sigma)$. For technical reasons that will become clear in Section 4 , we also require $\Phi_{s}(\sigma)$ to have the following properties:

(a) $\left|\Phi_{s}(\sigma)\right| \leq s$ (the approximation does not grow too quickly in length) and

(b) $\left|\Phi_{s+1}(\sigma \frown i)\right| \leq\left|\Phi_{s}(\sigma)\right|+1$, for any $i \in\{0,1\}$ (one more unit of time and one more bit of information will yield at most one additional bit of output).

If, for a real $x, \lim _{n}|\Phi(x \nmid n)|=\infty$, then $\Phi(x)=y$, where $y$ is the unique real that extends all $\Phi(x\lceil n)$. In this way, $\Phi$ also induces a partial, continuous function from $2^{\omega}$ to $2^{\omega}$. We will use the same symbol $\Phi$ for the Turing functional, the monotone function from $2^{<\omega}$ to $2^{<\omega}$, and the partial, continuous function from $2^{\omega}$ to $2^{\omega}$. It will be clear from the context which $\Phi$ is meant.

A Turing functional $\Phi$ has computably bounded use if there exists a computable function $g: \mathbb{N} \rightarrow \mathbb{N}$ so that $(m, k, \sigma) \in \Phi$ implies that $|\sigma| \leq g(m)$. If $\Phi(x)=y$ for such a functional, we say that $y$ is bounded Turing or weak truth-table reducible to $x, y \leq_{\mathrm{wtt}} x$.

Turing functionals can be relativized with respect to a parameter $z$ by requiring that $\Phi$ is c.e. in $z$. We call such functionals Turing $z$-functionals. This way we can consider relativized Turing reductions. A real $x$ is Turing reducible to a real $y$ relative to a real $z$, written $x \leq_{\mathrm{T}(z)} y$, if there exists a Turing $z$-functional $\Phi$ such that $\Phi(x)=y$.

2.3. Probability measures. A measure on $2^{\omega}$ is a countably additive, monotone function $\mu: \mathcal{F} \rightarrow[0,1]$, where $\mathcal{F} \subseteq \mathcal{P}\left(2^{\omega}\right)$ is a $\sigma$-algebra. If $\mu$ is normalized, i.e. if $\mu\left(2^{\omega}\right)=1$, then $\mu$ is called a probability measure. A measure $\mu$ is a Borel measure if $\mathcal{F}$ is the Borel $\sigma$-algebra on $2^{\omega}$. It is a basic result of measure theory that a measure with domain $\mathcal{F}$ is uniquely determined by the values it takes on an algebra $\mathcal{A} \subseteq \mathcal{F}$ that generates $\mathcal{F}$. It is not hard to see that in $2^{\omega}$, the Borel sets are generated by the algebra of clopen sets, i.e. finite unions of basic open cylinders. Normalized, countably additive, monotone set functions on the algebra of clopen sets are induced by any function $\rho: 2^{<\omega} \rightarrow[0,1]$ satisfying

$$
\rho(\epsilon)=1 \text { and for all } \sigma \in 2^{<\omega}, \rho(\sigma)=\rho(\sigma \frown 0)+\rho\left(\sigma^{\frown} 1\right),
$$

where $\epsilon$ denotes the empty string. If $\rho$ is as in (2.1), then putting $\mu(\llbracket \sigma \rrbracket)=\rho(\sigma)$ induces a monotone, additive function on the clopen sets, which in turn uniquely 
extends to a Borel probability measure on $2^{\omega}$. In the following, we will deal exclusively with Borel measures. Thus, when we speak of measures, we will always mean Borel measures. If convenient, we write $\mu A$ for $\mu(A)$ to improve readability.

The Lebesgue measure $\lambda$ on $2^{\omega}$ is obtained by distributing a unit mass uniformly along the paths of $2^{\omega}$, i.e. by setting $\lambda(\sigma)=2^{-|\sigma|}$. A Dirac measure, on the other hand, is defined by putting a unit mass on a single real; i.e. for $x \in 2^{\omega}$, let

$$
\delta_{x} \llbracket \sigma \rrbracket= \begin{cases}1 & \text { if } \sigma \sqsubset x, \\ 0 & \text { otherwise. }\end{cases}
$$

If, for a measure $\mu$ and $x \in 2^{\omega}, \mu\{x\}>0$, then $x$ is called an atom of $\mu$. Obviously, $x$ is an atom of $\delta_{x}$. A measure that does not have any atoms is called continuous.

2.4. The space of probability measures on Cantor space. We denote by $\mathcal{P}\left(2^{\omega}\right)$ the set of all probability measures on $2^{\omega} \cdot \mathcal{P}\left(2^{\omega}\right)$ can be given a topology (the so-called weak-* topology) by letting $\mu_{n} \rightarrow \mu$ if $\int f d \mu_{n} \rightarrow \int f d \mu$ for all continuous real-valued functions $f$ on $2^{\omega}$.

It is known that if $X$ is compact metrizable, then so is the space of all probability measures on $X$ (see for instance [12]). Therefore, $\mathcal{P}\left(2^{\omega}\right)$ is compact metrizable. In particular, it is Polish. A compatible metric (see for example [8]) is given by

$$
d_{\text {meas }}(\mu, \nu)=\sum_{n=1}^{\infty} 2^{-n} d_{n}(\mu, \nu),
$$

where

$$
d_{n}(\mu, \nu)=\frac{1}{2} \sum_{|\sigma|=n}|\mu \llbracket \sigma \rrbracket-\nu \llbracket \sigma \rrbracket| .
$$

A countable, dense subset $\mathcal{D} \subseteq \mathcal{P}\left(2^{\omega}\right)$ is given by the set of measures which assume positive, dyadic rational values (of the form $m / 2^{n}$ with $m, n \geq 0$ ) on a finite number of dyadic rationals; i.e. $\mathcal{D}$ is the set of measures of the form

$$
\nu_{\Delta, Q}=\sum_{\sigma \in \Delta} Q(\sigma) \delta_{\sigma \frown 0^{\omega}},
$$

where $\Delta$ is a finite set of finite strings (representing dyadic rational numbers) and $Q: \Delta \rightarrow[0,1]$ such that $\sum_{\sigma \in \Delta} Q(\sigma)=1$ and for all $\sigma \in \Delta, Q(\sigma)$ is a dyadic rational number.

It is straightforward to show that in case $\mu, \nu$ are restricted to measures in $\mathcal{D}$, the following relations are computable:

$$
d_{\text {meas }}(\mu, \nu)<q \quad \text { and } \quad d_{\text {meas }}(\mu, \nu) \leq q \quad(q \in \mathbb{Q} \cap[0,1]) .
$$

By effectively enumerating all possible combinations $(\Delta, Q)$, we can also effectively enumerate the set $\mathcal{D}$. In the following, we fix such an enumeration $\mathcal{D}=$ $\left\{\nu_{0}, \nu_{1}, \nu_{2}, \ldots\right\}$. The triple $\left(\mathcal{P}\left(2^{\omega}\right), \mathcal{D}, d_{\text {meas }}\right)$ forms a computable metric space (see e.g. 31] or [10, [7] in this particular context).

We can represent measures in $\mathcal{P}\left(2^{\omega}\right)$ through Cauchy sequences of measures in $\mathcal{D}$, which in turn can be encoded as reals using the enumeration of $\mathcal{D}$. Furthermore, the fact that $\mathcal{P}\left(2^{\omega}\right)$ is a compact, computable metric space can be used to devise a coding scheme that captures the topology of $\mathcal{P}\left(2^{\omega}\right)$. 


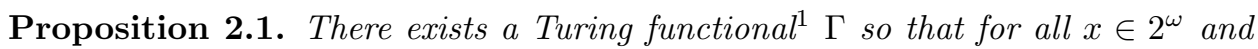
all $n \in \mathbb{N}, \Gamma^{x}(n)$ is defined and

$$
d_{\text {meas }}\left(\nu_{\Gamma^{x}(n)}, \nu_{\Gamma^{x}(n+1)}\right) \leq 2^{-n} .
$$

Furthermore, $\Gamma$ induces a continuous surjection $\rho: 2^{\omega} \rightarrow \mathcal{P}\left(2^{\omega}\right)$ by letting

$$
\rho(x)=\lim _{n} \nu_{\Gamma^{x}(n)},
$$

where the limit is taken with respect to the weak-* topology. Finally, $\rho$ is such that for any $x \in 2^{\omega}$, the set

$$
\rho^{-1}(\{\rho(x)\})
$$

is $\Pi_{1}^{0}(x)$.

For a proof, see [3]. (25] has a similar development of representations of measures.) If $\mu \in \mathcal{P}\left(2^{\omega}\right)$, any real $r$ with $\rho(r)=\mu$ is called a representation of $\mu$. Note that the representation of a measure is not unique. In fact, a measure may have uncountably many distinct representations.

Proposition 2.2. Let $r \in 2^{\omega}$ be a representation of a measure $\mu \in \mathcal{P}\left(2^{\omega}\right)$. Then the relations

$$
\mu \llbracket \sigma \rrbracket<q \quad \text { and } \quad \mu \llbracket \sigma \rrbracket>q \quad\left(\sigma \in 2^{<\omega}, q \in \mathbb{Q}\right)
$$

are c.e. in $r$.

Proof. Let $\gamma(k)=\Gamma^{r}(k)$, where $\Gamma$ is the Turing functional from Proposition 2.1. Since $d_{\text {meas }}\left(\nu_{\gamma(k)}, \mu\right) \leq 2^{-n+1}$, it follows from the definition of $d_{\text {meas }}$ that

$$
\left|\nu_{\gamma(k)} \llbracket \sigma \rrbracket-\mu \llbracket \sigma \rrbracket\right| \leq 2^{-k+|\sigma|+2} .
$$

This in turn implies

$$
\mu \llbracket \sigma \rrbracket \leq \nu_{\gamma(k)} \llbracket \sigma \rrbracket+2^{-k+|\sigma|+2} \leq \mu \llbracket \sigma \rrbracket+2^{-k+|\sigma|+3} .
$$

Hence $\mu \llbracket \sigma \rrbracket<q$ if and only if

$$
\exists k \nu_{\gamma(k)} \llbracket \sigma \rrbracket+2^{-k+|\sigma|+2}<q .
$$

But $\nu_{\gamma(k)} \llbracket \sigma \rrbracket$ is a dyadic rational uniformly computable in $r$, and hence $\nu_{\gamma(k)} \llbracket \sigma \rrbracket+$ $2^{-k+|\sigma|+2}<q$ is decidable given $r$ as an oracle. The proof for $\mu \llbracket \sigma \rrbracket>q$ is symmetrical.

Proposition 2.2 easily implies the following.

Proposition 2.3. Let $r \in 2^{\omega}$ be a representation of a measure $\mu \in \mathcal{P}\left(2^{\omega}\right)$. Then $r$ computes a function $g_{\mu}: 2^{<\omega} \times \mathbb{N} \rightarrow \mathbb{Q}$ such that for all $\sigma \in 2^{<\omega}, n \in \mathbb{N}$,

$$
\left|g_{\mu}(\sigma, n)-\mu \llbracket \sigma \rrbracket\right| \leq 2^{-n} .
$$

\footnotetext{
${ }^{1}$ The way we defined Turing functionals in Section 2.2 they induce partial mappings from $2^{\omega}$ to $2^{\omega}$. Here we obviously assume that $\Gamma$ induces a mapping from $2^{\omega}$ to $\omega^{\omega}$. The definition given in Section 2.2 is easily adjustable to this case.
} 


\section{RANDOMNESS AND TRANSFORMATIONS OF MEASURES}

3.1. Random reals. We define (relative) randomness of reals for arbitrary measures as a straightforward extension of Martin-Löf's test notion. The basic idea is to require the test to be enumerable $\left(\Sigma_{1}^{0}\right)$ in a representation of the measure.

Definition 3.1. Let $r_{\mu}$ be a representation of a measure $\mu$, and let $z \in 2^{\omega}$.

(a) An $\left(r_{\mu}, z\right)$-test is given by a sequence $\left(V_{n}: n \in \mathbb{N}\right)$ of uniformly $\Sigma_{1}^{0}\left(r_{\mu} \oplus z\right)$-sets $V_{n} \subseteq 2^{<\omega}$ such that for all $n$,

$$
\sum_{\sigma \in V_{n}} \mu \llbracket \sigma \rrbracket \leq 2^{-n} .
$$

(b) A real $x \in 2^{\omega}$ passes an $\left(r_{\mu}, z\right)$-test $\left(V_{n}\right)$ if $x \notin \bigcap_{n} \llbracket V_{n} \rrbracket$. Otherwise we say the test $\left(V_{n}\right)$ covers $x$.

(c) A real $x \in 2^{\omega}$ is $\left(r_{\mu}, z\right)$-random if it passes all $\left(r_{\mu}, z\right)$-tests.

If, in the previous definition, $z=\emptyset$ (where we identify reals with subsets of natural numbers via the characteristic sequence), we simply speak of an $r_{\mu}$-test and of $x$ being $r_{\mu}$-random.

The previous definition defines randomness with respect to a specific representation. If $x$ is random for one representation, it is not necessarily random for other representations. On the other hand, we can ask whether a real exhibits randomness with respect to some representation, so the following definition makes sense.

Definition 3.2. A real $x \in 2^{\omega}$ is $\mu$-random relative to $z \in 2^{\omega}$, or simply $\mu-z$ random, if there exists a representation $r_{\mu}$ of $\mu$ so that $x$ is $\left(r_{\mu}, z\right)$-random.

One might argue that this definition of randomness is subject to a certain arbitrariness, as it depends on a particular representation of a measure. However, it has recently been shown by Day and Miller 3 . that the definition of randomness given in Definition 3.2 is equivalent to the representation-independent approach via uniform tests due to Levin [16 18] and Gács [7].

Moreover, in this paper we are interested in results of the type "For which reals does there exist a measure for which $x$ looks non-trivially random?"; i.e. can $x$ look random at all? If there exists such a (representation of a) measure, there is good reason to say that $x$ has some random content. It is not the aim of this paper to provide a most general solution to the problem of defining randomness for arbitrary measures. The goal is to exhibit an interesting connection between the randomness properties of reals (with respect to some representation) and its logical complexity.

Of course, every real $x$ is trivially $\mu$-random if it is a $\mu$-atom. The question is, under what circumstances is $x$ non-trivially $\mu$-random, i.e. when does there exist a measure $\mu$ so that $x$ is $\mu$-random and $\mu\{x\}=0$ ?

A most useful property of the theory of Martin-Löf randomness is the existence of universal tests. Universal tests subsume all other tests. Furthermore, they can be defined uniformly with respect to any parameter. More precisely, for any representation $r_{\mu}$ of a measure $\mu$, there exists a uniformly c.e. in $r_{\mu}$ sequence $\left(U_{n}: n \in \mathbb{N}\right)$ of sets $U_{n} \subseteq 2^{<\omega}$ such that if we set for $z \in 2^{\omega}$,

$$
U_{n}^{z}=\left\{\sigma:\langle\sigma, \tau\rangle \in U_{n}, \tau \sqsubset z\right\},
$$

then $\left(U_{n}^{z}\right)$ is an $\left(r_{\mu}, z\right)$-test and $x \in 2^{\omega}$ is $\left(r_{\mu}, z\right)$-random if and only if $x$ passes $\left(U_{n}^{z}\right)$. We call $\left(U_{n}\right)$ a universal oracle test for $r_{\mu}$. For details on the existence of universal tests, see 2]. One can also construct universal tests that have a larger 
number of parameters. In Section 4 we will need a test that is universal for two parameters. Such a test is a uniformly c.e. in $r_{\mu}$ sequence $\left(U_{n}^{(2)}: n \in \mathbb{N}\right)$ so that if we set for $z_{0}, z_{1} \in 2^{\omega}$,

$$
U_{n}^{z_{0}, z_{1}}=\left\{\sigma:\left\langle\sigma, \tau_{0}, \tau_{1}\right\rangle \in U_{n}^{(2)}, \tau_{0} \sqsubset z_{0}, \tau_{1} \sqsubset z_{1}\right\},
$$

then $\left(U_{n}^{z_{0}, z_{1}}\right)$ is an $\left(r_{\mu}, z_{0} \oplus z_{1}\right)$-test and $x \in 2^{\omega}$ is $\left(r_{\mu}, z_{0} \oplus z_{1}\right)$-random if and only if $x$ passes $\left(U_{n}^{z_{0}, z_{1}}\right)$.

3.2. Computable measures. A measure is computable if there exists a computable function $g: 2^{<\omega} \times \mathbb{N} \rightarrow \mathbb{Q}$ such that for all $\sigma \in 2^{<\omega}, n \in \mathbb{N}$,

$$
|g(\sigma, n)-\mu \llbracket \sigma \rrbracket| \leq 2^{-n} .
$$

By Proposition 2.3. any measure with a computable representation is computable. The converse holds too (see [10]). For a computable measure $\mu$, not being $\mu-z$ random is equivalent to the existence of a sequence $\left(V_{n}\right)$ uniformly c.e. in $z$ so that for all $n, \mu \llbracket V_{n} \rrbracket \leq 2^{-n}$ and so that $\left(V_{n}\right)$ covers $x$. We call the latter a $(\mu, z)$-test.

Lebesgue measure $\lambda$ is computable, and it is arguably the most prominent measure on $2^{\omega}$. $\lambda$-random reals are the most studied ones by far, and we will, in consistency with the literature, use the name Martin-Lö random reals for them.

Reals that are random with respect to some computable probability measure have been called proper [33] or natural [22].

Obviously no computable real can be non-trivially random with respect to any measure. The following observation yields that the trivially random reals with respect to computable measures are precisely the computable reals.

Proposition 3.3 (Levin [33]). If $\mu$ is a computable measure and $\mu\{x\}>0$ for some $x \in 2^{\omega}$, then $x$ is computable.

Proof. Suppose $\mu\{x\}>2^{-m}>0$ for some computable $\mu$ and $m \geq 1$. Let $g$ be a computation function for $\mu$; i.e. $g$ is computable and for all $\sigma$ and $n, \mid g(\sigma, n)-$ $\mu \llbracket \sigma \rrbracket \mid \leq 2^{-n}$. Define a computable tree $T \subseteq 2^{<\omega}$ by letting $\sigma \in T$ if and only if $g(\sigma,|\sigma|) \geq 2^{-m}-2^{-|\sigma|} . x$ is an infinite path through $T$. We claim that $x$ is a fully isolated path; i.e. there exists a string $\sigma$ such that for all $\tau \sqsupseteq \sigma, \tau \in T$ implies $\tau \sqsubset x$. Clearly any fully isolated path through $T$ is computable.

Suppose $x$ were not fully isolated. Then there exist infinitely many $\sigma_{n} \sqsubset x$, $\sigma_{n} \sqsubset \sigma_{n+1}$ such that $\sigma_{n}^{\vee} \in T$, where $\sigma_{n}^{\vee}$ is obtained from $\sigma_{n}$ by switching the last bit. It follows that the $\sigma_{n}^{\vee}$ are pairwise incompatible. Since $\sigma_{n}^{\vee} \in T$, we have that for sufficiently large $n$,

$$
\mu \llbracket \sigma_{n}^{\vee} \rrbracket \geq \frac{1}{2^{m+1}},
$$

which is impossible since the $\sigma_{n}^{\vee}$ are pairwise incompatible and $\mu$ is a probability measure.

As regards non-computable reals, Levin proved that, from a computability theoretic point of view, randomness with respect to a computable probability measure is computationally as powerful as Martin-Löf randomness. This was independently shown by Kautz [11.

Theorem 3.4 (Levin [33], Kautz [1]). A non-computable real which is random with respect to some computable measure is Turing equivalent to a Martin-Löf random real. 
The proof of Theorem 3.4 uses the fact that reductions induce continuous (partial) mappings from $2^{\omega}$ to $2^{\omega}$. Such mappings transform measures.

3.3. Transformation of measures. Let $\mu$ be a Borel measure on $2^{\omega}$, and let $f: 2^{\omega} \rightarrow 2^{\omega}$ be a $\mu$-measurable function; that is, for all measurable $A \subseteq 2^{\omega}$, $f^{-1}(A)$ is $\mu$-measurable too. Such $f$ induces a new measure $\mu_{f}$, often referred to as the image measure or push-forward of $\mu$, on $2^{\omega}$ by letting

$$
\mu_{f}(A)=\mu\left(f^{-1}(A)\right) .
$$

Any non-atomic measure can be transformed into Lebesgue measure $\lambda$ this way. Recall that a function $f: 2^{\omega} \rightarrow 2^{\omega}$ is a Borel automorphism if it is bijective and for any $A \subseteq 2^{\omega}, A$ is a Borel set if and only if $f(A)$ is.

Theorem 3.5 (see [9]). Let $\mu$ be a non-atomic probability measure on $2^{\omega}$.

(1) There exists a continuous mapping $f: 2^{\omega} \rightarrow 2^{\omega}$ such that $\mu_{f}=\lambda$.

(2) There exists a Borel automorphism $h$ of $2^{\omega}$ such that $\mu_{h}=\lambda$.

The simple idea to prove (1) is to identify Cantor space with the unit interval $[0,1]$ and define $f(x)=\mu([0, x])$, that is, to let $f$ equal the distribution function of $\mu$.

To prove Theorem 3.4 Levin showed that this idea works in the effective case too. Furthermore, one can even deal with the presence of atoms, as long as the measure is computable. Roughly speaking, a computable transformation transforms a computable measure into another computable measure. At the same time, a computable transformation will preserve randomness in the sense that a real random with respect to $\mu$, when transformed by a computable mapping, is random with respect to the image measure of $\mu$.

In the following, we will use a (relativized) transformation of Lebesgue measure to render a given real random.

\section{RANDOMNESS With RESPECT TO ARBITRARY MEASURES}

The Levin-Kautz result implies that the set of reals that are non-trivially random with respect to a computable measure are contained in the set of Martin-Löf random Turing degrees. But what about the reals non-trivially random with respect to an arbitrary measure? In this section we will show that these coincide with the noncomputable reals. Every non-computable real is non-trivially random with respect to some measure.

The proof of this result uses two important results from computability theory and algorithmic randomness, the Kučera-Gács Theorem and the Posner-Robinson Theorem.

We will need the Kučera-Gács Theorem in the following, relativized form.

Theorem 4.1 (Kučera [15], Gács [6]). Let $x, z \in 2^{\omega}$. There exists a real $y$ that is Martin-Löf random relative to $z$ such that

$$
x \leq_{\mathrm{wtt}(z)} y \leq_{\mathrm{wtt}(z)} x \oplus z^{\prime} .
$$

Theorem 4.2 (Posner and Robinson [24]). If $x \in 2^{\omega}$ is non-computable, then there is a $z \in 2^{\omega}$ such that $x \oplus z \geq_{\mathrm{T}} z^{\prime}$. 
Corollary 4.3. For every non-computable real $x$, there exist reals $y, z \in 2^{\omega}$ so that $y$ is Martin-Löf random relative to $z$ and

$$
x \leq_{\mathrm{wtt}(z)} y \quad \text { and } \quad y \leq_{\mathrm{T}(z)} x .
$$

The (relative) Turing equivalence to a random real allows for transforming Lebesgue measure $\lambda$ in a sufficiently controlled manner. More precisely, we can obtain a $\Pi_{1}^{0}$ class $M$ of representations of measures. Each measure represented in this class is a good candidate for a measure that renders $x$ random. We will use a compactness argument to show that at least one member $r_{\mu}$ of $M$ has the property that the Martin-Löf random real $y$ is still $\lambda$-random relative to $r_{\mu}$ (here $r_{\mu}$ is viewed as a real, not a measure). Then, $x$ has to be $r_{\mu}$-random, since otherwise an $r_{\mu}$-test could be effectively transformed into a Martin-Löf $\lambda$-test relative to $r_{\mu}$ which $y$ would fail.

We now state and prove the main result of this section.

Theorem 4.4. For any real $x \in 2^{\omega}$, the following are equivalent:

(i) There exists a probability measure $\mu$ such that $x$ is not a $\mu$-atom and $x$ is $\mu$-random.

(ii) $x$ is not computable.

Proof. (i) $\Rightarrow$ (ii): If $x$ is computable and $\mu$ is a measure with $\mu\{x\}=0$, then we can construct a $\mu$-test that covers $x$ by using $x$ and any representation of $\mu$ to search for an initial segment of $x$ whose measure is sufficiently small. More formally, given $n$, compute, using any representation $r_{\mu}$ of $\mu$ as an oracle, a length $l_{n}$ for which $\mu \llbracket x\left\lceil l_{n} \rrbracket<2^{-n}\right.$. Define a $\mu$-test $\left(V_{n}\right)$ by letting $V_{n}=\left\{x\left\lceil l_{n}\right\}\right.$.

(ii) $\Rightarrow$ (i): Let $x$ be a non-computable real. Using Corollary 4.3, we obtain a real $y$ which is Martin-Löf random relative to some $z \in 2^{\omega}$ and which is $\mathrm{T}(z)$-equivalent to $x$.

There are Turing $z$-functionals $\Phi$ and $\Psi$ such that

$$
\Phi(y)=x \quad \text { and } \quad \Psi(x)=y .
$$

We will use the functionals $\Phi$ and $\Psi$ to define a set of measures $M$. If $\Phi$ were total and invertible, there would be no problem defining the desired measure, as one could simply 'push forward' Lebesgue measure using $\Phi$. In our case we have to use $\Phi$ and $\Psi$ to control the measure. We are guaranteed that this will work locally, since $\Phi$ and $\Psi$ are mutual inverses when restricted to $x$ and $y$. Therefore, given a string $\sigma$ (a possible initial segment of $x$ ) we will single out strings which appear to be candidates for initial segments of an inverse real.

For any $\sigma$, let $\operatorname{Pre}^{*}(\sigma) \subseteq 2^{<\omega}$ be defined as

$$
\operatorname{Pre}^{*}(\sigma)=\left\{\tau \in 2^{<\omega}: \Phi(\tau) \sqsupseteq \sigma \quad \& \quad \Psi_{|\sigma|}(\sigma) \sqsubseteq \tau\right\} .
$$

We will need only the elements of Pre* that are minimal with respect to the prefix relation. Let

$$
\operatorname{Pre}(\sigma)=\left\{\tau \in \operatorname{Pre}^{*}(\sigma): \forall \tau^{\prime} \in \operatorname{Pre}^{*}(\sigma)\left(\tau, \tau^{\prime} \text { compatible } \rightarrow \tau \sqsubseteq \tau^{\prime}\right)\right\} .
$$

Note that $\operatorname{Pre}(\sigma)$ is uniformly c.e. in $z$, since we can approximate $\Phi(\tau)$ by longer and longer prefixes (the strings $\Phi_{s}(\tau)$ ), and we assume the reductions $\Phi, \Psi$ to be use monotone. 
To define a measure $\mu$ with respect to which $x$ is non-trivially random, we satisfy two requirements:

(1) The measure $\mu$ dominates the partial push-forward of Lebesgue measure induced by $\Phi$. This will help ensure that any Martin-Löf random real is mapped by $\Phi$ to a $\mu$-random real.

(2) The measure $\mu$ must not be atomic on $x$.

To meet these requirements, we restrict the values of $\mu$ in the following way:

$$
\lambda \llbracket \operatorname{Pre}(\sigma) \rrbracket \leq \mu \llbracket \sigma \rrbracket \leq \lambda \llbracket \Psi_{|\sigma|}(\sigma) \rrbracket .
$$

The first inequality ensures that (1) is met, whereas the second guarantees that $\mu$ is non-atomic on the domain of $\Psi$ (since if $\Psi(z)$ is defined, then $\lim _{s} \mid \Psi_{s}(z\lceil s) \mid=\infty$ and thus $\left.\left.\lambda \llbracket \Psi_{s}(z \uparrow s)\right) \rrbracket \rightarrow 0\right)$. If $\Psi(z)$ is undefined, then $\Psi_{s}(z \uparrow s)$ is constant from some point on and hence imposes a constant positive upper bound on all $\mu \llbracket z\lceil s \rrbracket$ from that point on.

Let $M \subseteq 2^{\omega}$ be the set of all representations of measures that satisfy (4.1). We show that $M$ is non-empty and $\Pi_{1}^{0}(z)$.

Claim. The set $M$ is not empty.

Proof. We exhibit a measure $\mu$ that respects all upper and lower bounds given by (4.1). Since every measure has a representation, this implies that $M$ is non-empty. We construct $\mu$ inductively on the basic open cylinders. Put $\mu \llbracket \epsilon \rrbracket=1$. Suppose $\mu \llbracket \sigma \rrbracket$ is given such that

$$
\lambda \llbracket \operatorname{Pre}(\sigma) \rrbracket \leq \mu \llbracket \sigma \rrbracket \leq \lambda \llbracket \Psi_{|\sigma|}(\sigma) \rrbracket .
$$

It follows from the definition of Pre that

$$
\operatorname{Pre}(\sigma \frown 0), \operatorname{Pre}(\sigma \frown 1) \subseteq \operatorname{Pre}(\sigma) \quad \text { and } \quad \operatorname{Pre}(\sigma \frown 0) \cap \operatorname{Pre}\left(\sigma^{\frown} 1\right)=\emptyset .
$$

Hence, since $\lambda$ is a measure,

$$
\lambda \llbracket \operatorname{Pre}\left(\sigma^{\frown} 0\right) \rrbracket+\lambda \llbracket \operatorname{Pre}\left(\sigma^{\frown} 1\right) \rrbracket \leq \lambda \llbracket \operatorname{Pre}(\sigma) \rrbracket .
$$

Furthermore, by the properties of the approximation $\Psi_{s}$ stated in Section 2.2, we have

$$
\lambda \llbracket \Psi_{|\sigma \frown 0|}(\sigma \frown 0) \rrbracket+\lambda \llbracket \Psi_{|\sigma \frown 1|}(\sigma \frown 1) \rrbracket \geq 2^{-\left|\Psi_{|\sigma|}(\sigma)\right|-1}+2^{-\left|\Psi_{|\sigma|}(\sigma)\right|-1}=\lambda \llbracket \Psi_{|\sigma|}(\sigma) \rrbracket .
$$

Thus,

$$
\lambda \llbracket \operatorname{Pre}(\sigma \frown 0) \rrbracket+\lambda \llbracket \operatorname{Pre}(\sigma \frown 1) \rrbracket \leq \mu \llbracket \sigma \rrbracket \leq \lambda \llbracket \Psi_{|\sigma \frown 0|}(\sigma \frown 0) \rrbracket+\lambda \llbracket \Psi_{|\sigma \frown 1|}\left(\sigma^{\frown} 1\right) \rrbracket .
$$

Since the mapping $\theta:[0,1] \times[0,1] \rightarrow \mathbb{R}$ given by

$$
\begin{aligned}
\theta(s, t)=\lambda \llbracket \operatorname{Pre}(\sigma \frown 0) \rrbracket+ & s\left(\lambda \llbracket \Psi_{|\sigma \frown 0|}(\sigma \frown 0) \rrbracket-\lambda \llbracket \operatorname{Pre}(\sigma \frown 0) \rrbracket\right) \\
& +\lambda \llbracket \operatorname{Pre}(\sigma \frown 1) \rrbracket+t\left(\lambda \llbracket \Psi_{|\sigma \frown 1|}\left(\sigma^{\frown} 1\right) \rrbracket-\lambda \llbracket \operatorname{Pre}(\sigma \frown 1) \rrbracket\right)
\end{aligned}
$$

is continuous, it follows from the intermediate value theorem that there exist $s_{0}, t_{0}$ such that $\theta\left(s_{0}, t_{0}\right)=\mu \llbracket \sigma \rrbracket$. Put

$$
\begin{aligned}
& \mu \llbracket \sigma^{\frown} 0 \rrbracket=\lambda \llbracket \operatorname{Pre}\left(\sigma^{\frown} 0\right) \rrbracket+s_{0}\left(\lambda \llbracket \Psi_{|\sigma \frown 0|}\left(\sigma^{\frown} 0\right) \rrbracket-\lambda \llbracket \operatorname{Pre}\left(\sigma^{\frown} 0\right) \rrbracket\right), \\
& \mu \llbracket \sigma^{\frown} 1 \rrbracket=\lambda \llbracket \operatorname{Pre}\left(\sigma^{\frown} 1\right) \rrbracket+t_{0}\left(\lambda \llbracket \Psi_{|\sigma \frown 1|}\left(\sigma^{\frown} 1\right) \rrbracket-\lambda \llbracket \operatorname{Pre}\left(\sigma^{\frown} 1\right) \rrbracket\right) .
\end{aligned}
$$


Claim. The set $M$ is $\Pi_{1}^{0}(z)$.

Proof. A representation $r$ is in $M$ if and only if

$$
\forall \sigma \lambda \llbracket \operatorname{Pre}(\sigma) \rrbracket \leq \rho(r) \llbracket \sigma \rrbracket \leq \lambda \llbracket \Psi_{|\sigma|}(\sigma) \rrbracket .
$$

It suffices to show that the relation $\lambda \llbracket \operatorname{Pre}(\sigma) \rrbracket \leq \rho(r) \llbracket \sigma \rrbracket \leq \lambda \llbracket \Psi_{|\sigma|}(\sigma) \rrbracket$ is uniformly $\Pi_{1}^{0}(z)$.

The set $\operatorname{Pre}(\sigma)$ is c.e. in $z$ (uniformly in $\sigma$ ), and thus the measure $\lambda \llbracket \operatorname{Pre}(\sigma) \rrbracket$ is left-enumerable in $z$. There exists a strictly increasing, computable sequence of dyadic rationals $\left(q_{n}\right)$ so that $q_{n} \rightarrow \lambda \llbracket \operatorname{Pre}(\sigma) \rrbracket$. We have that

$$
\rho(r) \llbracket \sigma \rrbracket<\lambda \llbracket \operatorname{Pre}(\sigma) \rrbracket \quad \Leftrightarrow \quad \exists n \rho(r) \llbracket \sigma \rrbracket<q_{n} .
$$

By Proposition 2.2, $\rho(r) \llbracket \sigma \rrbracket<q_{n}$ is $\Sigma_{1}^{0}(z)$. Hence $\rho(r) \llbracket \sigma \rrbracket<\operatorname{Pre}(\sigma)$ is $\Sigma_{1}^{0}(z)$ too. Proposition 2.2 also yields that the relation $\rho(r) \llbracket \sigma \rrbracket>\lambda \llbracket \Psi_{|\sigma|}(\sigma) \rrbracket$ is $\Sigma_{1}^{0}(z)$. Hence the relation $\lambda \llbracket \operatorname{Pre}(\sigma) \rrbracket \leq \rho(r) \llbracket \sigma \rrbracket \leq \lambda \llbracket \Psi_{|\sigma|}(\sigma) \rrbracket$ is $\Pi_{1}^{0}(z)$, as desired, and the argument above is uniform in $\sigma$.

Let $r_{\mu} \in M$. It follows from the definition of $M$ that $x$ is not an atom of $\mu$. Suppose $\left(V_{n}\right)$ is an $\left(r_{\mu}, z\right)$-test that covers $x$. For each $n$, let

$$
W_{n}=\left\{\operatorname{Pre}(\sigma): \sigma \in V_{n}\right\} .
$$

Then $W_{n}$ is uniformly $\Sigma_{1}^{0}\left(r_{\mu} \oplus z\right)$ since $\operatorname{Pre}(\sigma)$ is uniformly c.e. in $z$. Furthermore,

$$
\lambda \llbracket W_{n} \rrbracket \leq \sum_{\sigma \in V_{n}} \lambda \llbracket \operatorname{Pre}(\sigma) \rrbracket \leq \sum_{\sigma \in V_{n}} \mu \llbracket \sigma \rrbracket \leq 2^{-n} .
$$

Hence $\left(W_{n}\right)$ is a $\left(\lambda, r_{\mu} \oplus z\right)$-test. Moreover, since $y \in \llbracket \operatorname{Pre}\left(x\lceil k) \rrbracket\right.$ for all $k$ and $\left(V_{n}\right)$ covers $x,\left(W_{n}\right)$ covers $y$.

At this point, we are not yet quite able to derive a contradiction, since we only have that $y$ is Martin-Löf random relative to $z$ and not necessarily relative to $r_{\mu} \oplus z$. However, we can use the following basis theorem for $\Pi_{1}^{0}$-classes to infer the existence of an element $r \in M$ so that $y$ is $(\lambda, r \oplus z)$-random. Lemma 4.5 was independently obtained by Downey, Hirschfeldt, Miller, and Nies [4].

Lemma 4.5. Let $z \in 2^{\omega}$, and let $T \subseteq 2^{<\omega}$ be a tree computable in $z$ such that $T$ has an infinite path. Then, for every real $y$ that is Martin-Löf random relative to $z$, there is an infinite path $v$ through $T$ such that $y$ is Martin-Löf random relative to $z \oplus v$.

Proof. We use the universal oracle test $\left(U_{n}^{(2)}\right)$ introduced in Section 3 . Given $z \in 2^{\omega}$ and $\tau \in 2^{<\omega}$, let

$$
U_{n}^{z, \tau}=\left\{\sigma:\left\langle\sigma, \tau_{0}, \tau_{1}\right\rangle \in U_{n}^{(2)}, \tau_{0} \sqsubset z, \tau_{1} \sqsubseteq \tau\right\},
$$

where $\left(U_{n}^{(2)}\right)$ is a universal $\lambda$-test for two parameters, as introduced in Section 3.1 . Note that the sequence $\left(U_{n}^{z, \sigma}\right)$ is uniformly c.e. in $z$ and forms a $(\lambda, z)$-test.

We enumerate a $(\lambda, z)$-test $\left(V_{n}\right)$ as follows: enumerate a string $\sigma$ into $V_{n}$ if $\llbracket \sigma \rrbracket$ is contained in $\llbracket U_{n}^{z, \tau} \rrbracket$ for all $\tau \in T$ with $|\tau|=|\sigma|$ (note that there are only finitely many $\operatorname{such} \tau)$.

If $y$ is Martin-Löf random relative to $z$, there has to be some $n$ such that $y \notin \llbracket V_{n} \rrbracket$. Let

$$
T_{0}=\left\{\tau \in T: \llbracket y \uparrow|\tau| \rrbracket \text { is not contained in } \llbracket U_{n}^{z, \tau} \rrbracket\right\} .
$$

$T_{0}$ is clearly closed under initial segments; hence it is a subtree of $T$. For every $m$, $y \uparrow m$ is not enumerated in $V_{n}$, which implies that $T_{0}$ has nodes of every length; in 
particular it is infinite. Applying König's Lemma yields an infinite path $v$ through $T_{0}$.

Now, $y$ is Martin-Löf random relative to $z \oplus v$. Suppose not; then, since $\left(U_{n}^{(2)}\right)$ is a universal oracle test for $\lambda, y \in \bigcap_{k} \llbracket U_{k}^{z, v} \rrbracket$. In particular, $y \in \llbracket U_{n}^{z, v} \rrbracket$. By the use principle, there is an initial segment $\tau \sqsubset v$ such that $y \in \llbracket U_{n}^{z, \tau} \rrbracket$, contradicting the fact that $\tau \in T_{0}$.

Using Lemma 4.5, we obtain a representation $r_{\mu} \in M$ so that $x$ is $\left(r_{\mu}, z\right)$-random. To complete the proof of Theorem 4.4, note that any $\left(r_{\mu}, z\right)$-random real is also $r_{\mu}$-random, and hence $\mu$-random.

How hard is it to find a measure that makes a given real $x$ random? The tree determining $M$ is computable in the parameter $z$, stemming from the PosnerRobinson Theorem. $z$ can be found recursively in $x \oplus \emptyset^{\prime}$. It takes another jump to find a path through the tree which conserves randomness for some $y$ Turing $z$-equivalent to $x$. Hence, there is a representation $r_{\mu} \leq_{\mathrm{T}} x^{\prime \prime}$ of a measure $\mu$ so that $x$ is $r_{\mu}$-random.

\section{RANDOMNESS With RESPECT to CONTINUOUS MEASURES}

In this section we investigate what happens if one replaces the property random with respect to an arbitrary probability measure (which turned out to hold for any non-computable real) with being random for a continuous probability measure.

First, we give an explicit construction of a non-computable real which does not have the latter property.

\subsection{A real not continuously random.}

Theorem 5.1. There exists a non-computable real which is not random with respect to any continuous measure.

Proof. Consider the halting problem $\emptyset^{\prime}$. Denote by $\emptyset_{t}^{\prime}$ the approximation to $\emptyset^{\prime}$ enumerated after $t$ steps. We define a set of markers $\gamma_{t}(n)$ that capture when the first $n$ bits of $\emptyset_{t}^{\prime}$ have settled. For each $t$, let $\gamma_{t}(0)=0$ and define

$$
\gamma_{t}(n+1):=\max \left\{\min \left\{s \leq t: \emptyset_{s}^{\prime}\left\lceil n+1=\emptyset_{t}^{\prime} \uparrow n+1\right\}, \gamma_{t}(n)+1\right\} .\right.
$$

Each $\gamma_{t}(n)$ (as a function of $t$ ) will be constant from some point on as computations of $\emptyset^{\prime}$ settle. We denote this value by $\gamma(n)$. Let $y_{t}$ be the real given by the characteristic sequence of $\left\{\gamma_{t}(n): n \geq 0\right\}$, and let $y$ be the real given by the characteristic sequence of $\{\gamma(n): n \geq 0\}$. We claim that $y$ is not random with respect to any continuous measure.

Let $\mu \in \mathcal{P}\left(2^{\omega}\right)$ be continuous, and let $r_{\mu}$ be any representation of $\mu$. Since $2^{\omega}$ is compact and $\mu$ is continuous, for every rational $\varepsilon>0$ there exists a number $l(\varepsilon)$ such that

$$
\forall \sigma \in\{0,1\}^{l(\varepsilon)} \mu \llbracket \sigma \rrbracket<\varepsilon .
$$

By Proposition 2.2 , the relation $\mu \llbracket \sigma \rrbracket<\varepsilon$ is $\Sigma_{1}^{0}\left(r_{\mu}\right)$. Therefore, $r_{\mu}$ can compute such a function $l$. We use this to uniformly enumerate the $n$th level of an $r_{\mu}$-test $\left(V_{n}\right)_{n \in \mathbb{N}}$ that covers $y$. 
First, compute $n_{0}=l\left(2^{-n-1}\right)$ and $n_{1}=l\left(2^{-n-1} / n_{0}\right)$. Enumerate $y_{n_{1}} \uparrow n_{0}$ into $V_{n}$. Furthermore, for all $k$ such that $\gamma_{n_{1}}(k)<n_{0}$, enumerate the string

$$
\left(y_{n_{1}} \uparrow \gamma_{n_{1}}(k)\right) \frown 0^{n_{1}-\gamma_{n_{1}}(k)}
$$

into $V_{n}$.

Note that $y_{t} \uparrow n_{0}$ can change at most $n_{0}$ many times. Observe further that if the approximation to $\emptyset^{\prime}$ changes at a position $m$ at time $t$, it will move the marker $\gamma_{t}(m)$ and with it all the markers $\gamma_{t}(k), k>m$, to a position $\geq t$. Hence, for the maximum $k \leq n_{0}$ so that $\gamma_{n_{1}}(k)=\gamma(k)$,

$$
y_{n_{1}}\left\lceil\gamma_{n_{1}}(k)=y\left\lceil\gamma_{n_{1}}(k)=y\lceil\gamma(k),\right.\right.
$$

and between $\gamma_{n_{1}}(k)$ and $n_{1}$ the characteristic sequence of $y$ has only 0 s. Therefore, $y$ is covered by $V_{n}$.

Finally, note that the measure of $V_{n}$ is at most $2^{-n}$, since

$$
\begin{aligned}
\sum_{v \in V_{n}} \mu \llbracket v \rrbracket & =\mu \llbracket y_{n_{1}}\left\lceil n_{0} \rrbracket+\sum_{\left\{k: \gamma_{n_{1}}(k)<n_{0}\right\}} \mu \llbracket\left(y_{n_{1}}\left\lceil\gamma_{n_{1}}(k)\right) \frown 0^{n_{1}-\gamma_{n_{1}}(k)} \rrbracket\right.\right. \\
& \leq 2^{-n-1}+n_{0} \frac{2^{-n-1}}{n_{0}}=2^{-n} .
\end{aligned}
$$

5.2. Classifying the not continuously random reals. Theorem 5.1 suggests the following question: Is it possible to classify the reals which are not random with respect to a continuous measure? Denote by NCR the set of all such reals. Can we obtain bounds on the complexity of NCR?

An observation by Kjos-Hanssen and Montalbán [13] shows that NCR is cofinal in the hyperarithmetical Turing degrees, i.e. for any hyperarithmetical $x$ there exists a hyperarithmetical $y \in \mathrm{NCR}$ so that $x \leq_{\mathrm{T}} y$.

It follows directly from the countable additivity of measures that for every continuous measure $\mu$, any countable subset of $2^{\omega}$ has $\mu$-measure zero.

For countable $\Pi_{1}^{0}$ classes, we can strengthen this to effective $\mu$-measure zero, and hence no countable $\Pi_{1}^{0}$ class contains a real in NCR.

Theorem 5.2 (Kjos-Hanssen and Montalbán [13]). If $A \subseteq 2^{\omega}$ is countable and $\Pi_{1}^{0}$, then no member of $A$ can be random with respect to a continuous measure.

Proof. Let $A=[T]$ for some computable tree $T$, and suppose $\mu$ is a continuous measure. Let $r_{\mu}$ be any representation of $\mu$. Let $T^{=n}$ denote the strings in $T$ of length $n$. It holds that

$$
\mu \llbracket T^{=n} \rrbracket \rightarrow \mu[T]=0 \quad(n \rightarrow \infty) .
$$

Using Proposition 2.2, we can browse the tree $T$ level by level till we see that the measure of $\llbracket T^{=n} \rrbracket$ falls below $2^{-n}$. When this happens, we enumerate all strings in $T^{=n}$ into the $n$th level of an $r_{\mu}$-test.

Kreisel [14] showed that every member of a countable $\Pi_{1}^{0}$ class is hyperarithmetic, i.e. contained in $\Delta_{1}^{1}$. Furthermore, he showed that members of countable $\Pi_{1}^{0}$ classes (also called ranked points) can be found cofinally in the hyperarithmetical Turing degrees. Later, Cenzer, Clote, Smith, Soare, and Wainer [1] showed that ranked points appear at each hyperarithmetical level of the Turing jump, i.e. can be found in Turing degrees obtained by iterating the Turing jump along a computable ordinal. 
Corollary 5.3. For every computable ordinal $\beta$ there exists an $x \in$ NCR such that $x \equiv_{\mathrm{T}} \emptyset^{(\beta)}$.

We would like to obtain an upper bound on the complexity of reals in NCR; in particular, we would like to know whether NCR is countable. We start with the following simple observation.

Proposition 5.4. The set NCR of all reals not random with respect to any continuous measure is $\Pi_{1}^{1}$.

Proof. We have

$x \in \mathrm{NCR} \Leftrightarrow(\forall r)[r$ represents a measure $\mu$ and $\mu$ is continuous

$$
\rightarrow \text { some } r \text {-test covers } x] \text {. }
$$

The property ' $r$ represents a measure' is obviously arithmetic, and due to compactness of $2^{\omega}$, a measure $\mu$ is continuous if and only if

$$
(\forall n)(\exists l)(\forall \sigma)\left[|\sigma|=l \rightarrow \mu \llbracket \sigma \rrbracket \leq 2^{-n}\right] .
$$

Hence ' $\mu$ is continuous' is arithmetic in $r$. Furthermore 'some test covers $x$ ' can be expressed as

$$
(\exists e)(\forall n)\left[\left[(\forall s) \sum_{\sigma \in W_{\{e\}_{s}^{r}(n)}} \rho(r) \llbracket \sigma \rrbracket \leq 2^{-n}\right] \wedge(\exists \sigma)\left[\sigma \in W_{\{e\}_{s}^{r}(n)} \wedge \sigma \sqsubset x\right]\right],
$$

which is arithmetic in $x$ and $r$.

Furthermore, it is not hard to see that NCR does not have a perfect subset.

Proposition 5.5. NCR does not have a perfect subset.

Proof. Assume $X \subseteq \mathrm{NCR}$ is a perfect subset represented by a perfect tree $T$ with $[T]=X$. We devise a measure $\mu$ by setting $\mu \llbracket \epsilon \rrbracket=1$, and define inductively

$$
\mu \llbracket \sigma \frown i \rrbracket= \begin{cases}\mu \llbracket \sigma \rrbracket & \text { if } \sigma \frown(1-i) \notin T, \\ \frac{1}{2} \mu \llbracket \sigma \rrbracket & \text { otherwise; }\end{cases}
$$

i.e. we distribute the measure uniformly over the infinite paths through $T$.

Obviously, $\mu$ is continuous, and since $\mu(X)=1, X$ must contain a $\mu$-random real. (The set of $\mu$-random reals is always a set of $\mu$-measure 1.)

The Perfect Subset Property refers to the principle that every set in a pointclass is either countable or contains a perfect subset. The Perfect Subset Property for $\boldsymbol{\Pi}_{1}^{1}$ is not provable in ZFC. Gödel showed that if $V=L$, then there exists an uncountable $\Pi_{1}^{1}$ set without a perfect subset. Mansfield [19] and Solovay [30] showed that any $\Sigma_{2}^{1}$ set without a perfect subset is contained in the constructible universe $L$.

Corollary 5.6. NCR is contained in Gödel's constructible universe L.

The upper bound $L$ appears indeed very crude, and an analysis of the proof technique of Theorem 4.4 together with a recent result by Woodin 32 will yield that NCR is countable. Even more, Corollary 5.3 is in a certain sense optimal: Every real outside $\Delta_{1}^{1}$ is random with respect to some continuous measure.

In the proof of Theorem 4.4, the decisive property which ensured the non-trivial $\mu$-randomness of $x$ was (4.1):

$$
\lambda \llbracket \operatorname{Pre}(\sigma) \rrbracket \leq \mu \llbracket \sigma \rrbracket \leq \lambda \llbracket \Psi_{|\sigma|}(\sigma) \rrbracket .
$$


Here, the second inequality guarantees that $x$ is not a $\mu$-atom. Although we know $\mu \llbracket \sigma \rrbracket$ will converge to 0 as we consider longer and longer initial segments $\sigma \sqsubset x$, this may not to be the case for reals other than $x$, as the reduction $\Psi$ from $x$ to $y$ is a Turing reduction.

If, however, $\Psi$ is a wtt-reduction, we can modify the construction in the proof of Theorem 4.4 to obtain a continuous measure with respect to which $x$ is random.

Theorem 5.7. Let $x \in 2^{\omega}$. Suppose there exist reals $y, z \in 2^{\omega}$ so that $y$ is MartinLöf random relative to $z$ and

$$
x \equiv_{\mathrm{wtt}(z)} y .
$$

Then $x$ is random with respect to a continuous measure.

Proof. The proof is similar to the proof of Theorem 4.4, with one important modification. Suppose $\Phi$ and $\Psi$ are wtt $z$-functionals such that

$$
\Phi(y)=x \quad \text { and } \quad \Psi(x)=y .
$$

(In fact, for the proof to work it suffices that $\Phi$ is a Turing $z$-functional.) Let $g: \mathbb{N} \rightarrow \mathbb{N}$ be a computable bound on the use of $\Psi$. Since both $x, y$ are noncomputable, we have that $g$ is unbounded. We may assume that $g$ is strictly decreasing. Define a computable function $h$ as

$$
h(k)=\max \{m: g(m) \leq k\} .
$$

If, for some $v \in 2^{\omega}, \Psi(v)$ is a real, then $\Psi(v \uparrow k)$ is a string of length at least $h(k)$ (for all $k$ ). Since $g$ is strictly increasing, we have that $h(k+1) \leq h(k)+1$.

Define the set $\operatorname{Pre}^{*}(\sigma)$ now as

$$
\operatorname{Pre}^{*}(\sigma)=\left\{\tau \in 2^{<\omega}: \Phi(\tau) \sqsupseteq \sigma \quad \& \quad \exists s\left(\left|\Psi_{s}(\sigma)\right| \geq h(|\sigma|) \& \Psi_{s}(\sigma) \sqsubseteq \tau\right)\right\} .
$$

As before, let Pre be the set of minimal elements of Pre* with respect to the prefix relation. Note that Pre is c.e. in $z$, as before.

Condition (4.1) is replaced by

$$
\lambda \llbracket \operatorname{Pre}(\sigma) \rrbracket \leq \mu \llbracket \sigma \rrbracket \leq 2^{-h(|\sigma|)},
$$

and $M$ is the set of all reals $r$ so that $\rho(r)$ satisfies (5.1) for all $\sigma \in 2^{<\omega}$.

As $h(k) \rightarrow \infty$ as $k \rightarrow \infty$, it follows that every measure represented in $M$ is continuous. The condition $h(k+1) \leq h(k)+1$ ensures that the proof showing $M$ is non-empty still goes through. A similar argument as in the proof of Theorem 4.4 shows that $M$ is $\Pi_{1}^{0}(z)$.

Finally, since $\Psi(x)=y, y \in \llbracket \operatorname{Pre}(x\lceil n) \rrbracket$ for all $n$. This in turn implies that the final part of the argument, transforming a possible test for $x$ into a test for $y$ (with respect to the accordant measures), goes through as well.

Woodin showed that outside the hyperarithmetical sets, the Posner-Robinson Theorem holds with truth-table equivalence.

Theorem 5.8 (Woodin [32]). If $x \in 2^{\omega}$ is not hyperarithmetic, then there is a $z \in 2^{\omega}$ such that $x \equiv_{\mathrm{tt}(z)} z^{\prime}$.

Combining Theorems 4.1, 5.8, and 5.7 now yields the desired upper bound for NCR.

Theorem 5.9. If a real $x$ is not hyperarithmetic, then there exists a continuous measure $\mu$ such that $x$ is $\mu$-random. 
Theorem 5.9 yields an interesting measure-theoretic characterization of $\Delta_{1}^{1}$. The result can also be obtained via a game-theoretic argument using Borel determinacy, along with a generalization of the Posner-Robinson Theorem via Kumabe-Slaman forcing. This is part of a more general argument which shows that for all $n$, the set $\mathrm{NCR}_{n}$ of reals which are not $n$-random for some continuous measure is countable. Here $n$-random means that a test has access to the $(n-1)$ st jump of a representation of the measure. The countability result for $\mathrm{NCR}_{n}$ has an interesting metamathematical twist. This work will be presented in a separate paper [26.

\section{ACKNOWLEDGMENT}

The authors thank an anonymous referee for many insightful and helpful comments and suggestions.

\section{REFERENCES}

[1] Douglas Cenzer, Peter Clote, Rick L. Smith, Robert I. Soare, and Stanley S. Wainer, Members of countable $\Pi_{1}^{0}$ classes: Special issue: second Southeast Asian logic conference (Bangkok, 1984), Ann. Pure Appl. Logic 31 (1986), no. 2-3, 145-163, DOI 10.1016/0168-0072(86)900679. MR854290 (88e:03064)

[2] A. Day, Randomness and computability, PhD thesis, Victoria University of Wellington, 2011.

[3] Adam R. Day and Joseph S. Miller, Randomness for non-computable measures, Trans. Amer. Math. Soc. 365 (2013), no. 7, 3575-3591, DOI 10.1090/S0002-9947-2013-05682-6. MR.3042595

[4] Rod Downey, Denis R. Hirschfeldt, Joseph S. Miller, and André Nies, Relativizing Chaitin's halting probability, J. Math. Log. 5 (2005), no. 2, 167-192, DOI 10.1142/S0219061305000468. MR2188515 (2007e:68031)

[5] Rodney G. Downey and Denis R. Hirschfeldt, Algorithmic randomness and complexity, Theory and Applications of Computability, Springer, New York, 2010. MR2732288 (2012g:03001)

[6] Péter Gács, Every sequence is reducible to a random one, Inform. and Control 70 (1986), no. 2-3, 186-192, DOI 10.1016/S0019-9958(86)80004-3. MR859105 (87k:03043)

[7] Peter Gács, Uniform test of algorithmic randomness over a general space, Theoret. Comput. Sci. 341 (2005), no. 1-3, 91-137, DOI 10.1016/j.tcs.2005.03.054. MR2159646 (2006m:68057)

[8] Eli Glasner, Ergodic theory via joinings, Mathematical Surveys and Monographs, vol. 101, American Mathematical Society, Providence, RI, 2003. MR.1958753 (2004c:37011)

[9] Paul R. Halmos, Measure theory, D. Van Nostrand Company, Inc., New York, N. Y., 1950. MR0033869(11,504d)

[10] Mathieu Hoyrup and Cristóbal Rojas, Computability of probability measures and MartinLöf randomness over metric spaces, Inform. and Comput. 207 (2009), no. 7, 830-847, DOI 10.1016/j.ic.2008.12.009. MR2519075 (2011b:03066)

[11] S. M. Kautz, Degrees of random sequences, PhD thesis, Cornell University, 1991.

[12] Alexander S. Kechris, Classical descriptive set theory, Graduate Texts in Mathematics, vol. 156, Springer-Verlag, New York, 1995. MR1321597(96e:03057)

[13] B. Kjos-Hanssen and A. Montalbán, Personal communication, March 2005.

[14] G. Kreisel, Analysis of the Cantor-Bendixson theorem by means of the analytic hierarchy (English, with Russian summary), Bull. Acad. Polon. Sci. Sér. Sci. Math. Astr. Phys. 7 (1959), 621-626 (unbound insert). MR0118673 (22 \#9444)

[15] Antonín Kučera, Measure, $\Pi_{1}^{0}$-classes and complete extensions of PA, Recursion theory week (Oberwolfach, 1984), Lecture Notes in Math., vol. 1141, Springer, Berlin, 1985, pp. 245-259, DOI 10.1007/BFb0076224. MR820784 (87e:03102)

[16] L. A. Levin, The concept of a random sequence (Russian), Dokl. Akad. Nauk SSSR 212 (1973), 548-550. MR0366096 (51 \#2346)

[17] L. A. Levin, Uniform tests for randomness (Russian), Dokl. Akad. Nauk SSSR 227 (1976), no. 1, 33-35. MR0414222 (54 \#2325)

[18] Leonid A. Levin, Randomness conservation inequalities: information and independence in mathematical theories, Inform. and Control 61 (1984), no. 1, 15-37, DOI 10.1016/S00199958(84)80060-1. MR764286 (86h:68081) 
[19] Richard Mansfield, Perfect subsets of definable sets of real numbers, Pacific J. Math. 35 (1970), 451-457. MR0280380 (43 \#6100)

[20] Per Martin-Löf, The definition of random sequences, Information and Control 9 (1966), 602619. MR0223179 (36 \#6228)

[21] Yiannis N. Moschovakis, Descriptive set theory, Studies in Logic and the Foundations of Mathematics, vol. 100, North-Holland Publishing Co., Amsterdam, 1980. MR561709 (82e:03002)

[22] Andrei A. Muchnik, Alexei L. Semenov, and Vladimir A. Uspensky, Mathematical metaphysics of randomness, Theoret. Comput. Sci. 207 (1998), no. 2, 263-317, DOI 10.1016/S0304-3975(98)00069-3. MR 1643438 (99h:68091)

[23] André Nies, Computability and randomness, Oxford Logic Guides, vol. 51, Oxford University Press, Oxford, 2009. MR2548883(2011i:03003)

[24] David B. Posner and Robert W. Robinson, Degrees joining to 0', J. Symbolic Logic 46 (1981), no. 4, 714-722, DOI 10.2307/2273221. MR641485 (83c:03040)

[25] Jan Reimann, Effectively closed sets of measures and randomness, Ann. Pure Appl. Logic 156 (2008), no. 1, 170-182, DOI 10.1016/j.apal.2008.06.015. MR2474448 (2010a:03043)

[26] J. Reimann and T. A. Slaman, Randomness for continuous measures, in preparation.

[27] Gerald E. Sacks, Degrees of unsolvability, Princeton University Press, Princeton, N.J., 1963. MR0186554 (32 \#4013)

[28] Claus-Peter Schnorr, Zufälligkeit und Wahrscheinlichkeit. Eine algorithmische Begründung der Wahrscheinlichkeitstheorie, Lecture Notes in Mathematics, Vol. 218, Springer-Verlag, Berlin-New York, 1971. MR0414225 (54 \#2328)

[29] Robert I. Soare, Recursively enumerable sets and degrees: A study of computable functions and computably generated sets, Perspectives in Mathematical Logic, Springer-Verlag, Berlin, 1987. MR882921 (88m:03003)

[30] Robert M. Solovay, On the cardinality of $\sum_{2}^{1}$ sets of reals, Foundations of Mathematics (Symposium Commemorating Kurt Gödel, Columbus, Ohio, 1966), Springer, New York, 1969, pp. 58-73. MR.0277382 (43 \#3115)

[31] Klaus Weihrauch, Computable analysis: An introduction, Texts in Theoretical Computer Science. An EATCS Series, Springer-Verlag, Berlin, 2000. MR1795407(2002b:03129)

[32] W. Hugh Woodin, A tt version of the Posner-Robinson theorem, Computational prospects of infinity. Part II. Presented talks, Lect. Notes Ser. Inst. Math. Sci. Natl. Univ. Singap., vol. 15, World Sci. Publ., Hackensack, NJ, 2008, pp. 355-392, DOI 10.1142/9789812796554_0019. MR2449474(2009j:03067)

[33] A. K. Zvonkin and L. A. Levin, The complexity of finite objects and the basing of the concepts of information and randomness on the theory of algorithms (Russian), Uspehi Mat. Nauk 25 (1970), no. 6(156), 85-127. MR.0307889 (46 \#7004)

Department of Mathematics, Pennsylvania State University, University Park, PennSYLVANIA 16802

E-mail address: reimann@math.psu.edu

Department of Mathematics, University of California at Berkeley, Berkeley, CalIFORNIA 94720

E-mail address: slaman@math.berkeley.edu 